\title{
Research and Implementation of Product Functional Design Based on Effect
}

\author{
Cao Guozhong, Tan Runhua and Lian Benning \\ School of Mechanical Engineering, Hebei University of Technology, \\ Tianjin, P.R. China \\ cgzghx@163.com
}

\begin{abstract}
Functional design is a process includes function understanding, function recognition, function representation, function modeling and functionto-structure mapping. The effect in TRIZ is extended by multi-pole effect model and effect chain modes, and then the functions and flows in TRIZ are reclassified. The combinative relationships among functions are discussed, and the function modeling based on effect is proposed. The principle structure is introduced, and the combined rules are presented. The functional design process model for function modeling and function-to-structure mapping is proposed, and the computer-aided functional design software is developed. A design example is presented to demonstrate the proposed functional design methodology.
\end{abstract}

Keywords. Functional Design, Effect, Principle Structure

\section{Introduction}

Functional design, which plays the central role in ensuring design quality and product innovation, is a well-researched and active field of engineering study.

There are various, often conflicting, definitions of function in the literature; no universally accepted definition is currently known, such as, designer's purpose [1,2], intended behavior [3], an effect on the environment of the product [4], a description of behavior recognized by a human through abstraction in order to utilize it [5] or a relationship between inputs and outputs, aiming to achieve the designer's purpose [6]. Clearly, each of these definitions has some aspects of worth, yet none are comprehensive enough to capture the fullness of definition that is desired.

Researchers have recognized the importance of a common vocabulary for broader issues of design. Pahl and Beitz [6] list five generally valid functions and three types of flows at a very high level of abstraction. Collins et al.[7] develop a list of 105 mechanical functions, which are limited to helicopter systems and do not utilize any classification scheme. TRIZ describes all mechanical design with a set of 30

Please use the following format when citing this chapter:

Guozhong, C., Runhua, T., Benning, L., 2007, in IFIP International Federation for Information Processing, Volume 250, Trends in Computer Aided Innovation, cd. Lcón-Rovira, N., (Boston: Springer), pp. 67-76. 
functional descriptions [8]. Malmqvist et al. [9] compare TRIZ with the Pahl and Beitz methodology and note that the detailed vocabulary of TRIZ would benefit from a more carefully structured class hierarchy using the Pahl and Beitz functions at the highest level.

Functional composition has been given considerable attention by researchers. Systematic design [6] characterizes an overall function can be adequately defined in terms of quantified inputs and outputs, and use input/output flow analysis and synthesis method to model a function structure. Axiomatic design [10] states that functional decomposition should be viewed as a zigzag mapping between functional requirements in a functional domain and design parameters in a physical domain. Struges et al. [11] present a scheme that is able to specify both vertical and horizontal functional relationships by functional block diagram. Chen [12] defines a general functional representation structure in terms of category, level and layer. Topdown decomposition methods provide a means for dividing a general function into sub-functions, but they can not describe the key characteristics of systems.

One of the most well-known functional design frameworks is that of Pahl \& Beitz [6], i.e., systematic approach, which model the overall function and decompose it into sub-functions operating on the flows of energy, material, and signals. Umeda et al. [13] proposed a Function-Behavior-State (FBS) modeler that reasons about function by means of two approaches: causal decomposition and task decomposition. Deng et al. [14] devised a dual-step function-environment-behaviour-structure (FEBS) model There are other similar approaches for functional models, for example, Qian and Gero's [1] FBS Path, and Prabhakar and Goel's [2] ESBF model.

During functional design the design knowledge and technologies in multiple different domains may be employed, and complicated developing activities. Although there are now some general methodologies dealing with functions or reasoning about functions, at present time, satisfying solution to both issues has not yet arrived [15].

Aiming at solving some crucial issues discussed above, this paper constructs a functional design process model for function modeling and function-to-structure mapping, which is based on multi-pole effect model, effect chain modes and combined rules of principle structure.

\section{Effect}

Effect is one of the knowledge base tools in TRIZ. By the analysis of hundreds of thousands patents, effects are emerged from the relevance between functions delivered by a design product described in a patent and a principle used in the product [16]. An Effects is an input and output relationship that combines the laws of science including physics, mathematics, chemistry and geometry, and their corresponding engineering applications, which helps to bridge the gap between science and engineering and is good for generating solutions of high levels. However, few literatures on effect exist in TRIZ monographs and few researches on effect exist in international, for example, only the concept, sort and use of effect are introduced in literature [17-19]. There is no literature on functional design based on effect. 


\section{$2.1 \quad$ Effect Model}

Effects can be characterized by its input, output relations [20]. An effect has an input and output flow, which is called as basic effect, thus the effect model has two poles [33], as shown in Fig. 1(a). The two-pole effect model is extended in this paper. Most transitions from input to output with effect are controlled by auxiliary flow, so the controllable effect should be denoted with three poles, as shown in Fig. 1(b). The control flow specifies the factors that can be manipulated to change the output intensity of an effect. Generally speaking, an effect may have multiple input flows, output flows or control flows, so the effect has multiple input poles, output poles or control poles, as shown in Fig. 1(c).

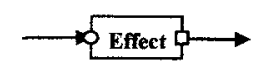

(a) Effect model with two poles

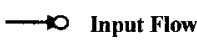

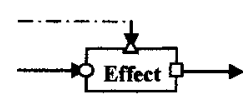

(b) Effect model with three poles

$\square$ Output Flow

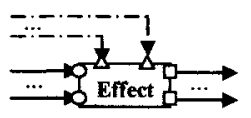

(c) Effect model with multi-pole

Fig. 1. Effect model

\subsection{Effect Chain and Effect Mode}

By the analysis of patents, the mapping relationship between function and effect is confirmed. The function fulfilled directly by effect is called as function unit. But, it is difficult for complex function to perform it by an effect. The effects need to be linked sequentially into chains through their input, output or control ports and compatibility of adjacent effects. Effect modes are the basic coupling manners of effect chains, as shown in the following:

- Serial effect mode: composed of several effects occurring in sequence, as shown in Fig. 2(a).

- Parallel effect mode: composed of several effects occurring at same time, as shown in Fig. 2(b).

- Ring effect mode: composed of several effects a set of effects which the input of a former effect is the output of a latter effect, as shown in Fig. 2(c).

- Control effect mode: the internal characteristic of an effect can be controlled by other effects in order to control output of the effect, as shown in Fig. 2(d).

- Combined effect mode: is composed of several above effects modes.

The effects can be linked into effect chain by using effect modes. During the transformation from input flow to output flow by effect modes, there are three methods, namely, method of exhaustion, method of minimal path length and method of consistent degree. The effect chain can help engineers achieve breakthrough innovation by proposing new and unexpected variations in producing a specific output. 


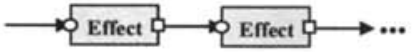

(a) Serial effect mode

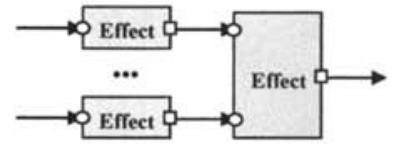

(b) Parallel effect mode

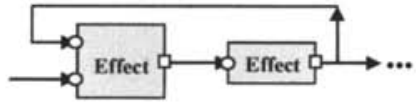

(c) Ring effect mode

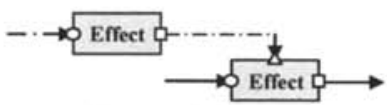

(d) Control effect mode

Fig. 2. Effect modes

\section{Function}

\subsection{Function Set}

Function is a statement to describe the transformation between inputs and outputs, aiming to achieve the designer's purpose. A function consists of commonly input/output part, and operation part. The input and output entities are referred to as functional flows. The operations are referred to as behaviors. Function is expressed as noun-verb-noun, which is different from the accustomed expression of verb-noun. Thus the function and effect have a consistent pattern of expression, and the horizontal interaction relationships among sub-functions can be confirmed and verified by compatibility of adjacent inputs and outputs.

Subsuming the classification schemes discussed above, the 30 functions in TRIZ are expanded and reclassified and the standard set of functions is presented, which includes a behavior (verb) set and a flow (noun) set, as shown in Table 1 and 2 .

Table 1. Short list of behavior set

\begin{tabular}{|l|l|}
\hline Behavior & Sub-behavior \\
\hline Create & Synthesize, Produce \\
\hline Change & Increase, Decrease, Convert, Deform, Control \\
\hline Combine & Mix, Embed, Assemble, connect \\
\hline Separate & Disassemble, Decompose, Dry, Clean \\
\hline Accumulate & Absorb, Store, Concentrate \\
\hline Move & Move, Transfer, Rotate, Vibrate, Lift, Orient \\
\hline Measure & Determine, Detect, Measure \\
\hline Preserve & Preserve, Prevent, Stabilize \\
\hline Eliminate & Destroy, Remove \\
\hline
\end{tabular}


Table 2. Short list of flow set

\begin{tabular}{|l|l|}
\hline Flow & Sub-flow \\
\hline Material & $\begin{array}{l}\text { Solid, Liquid, Gas, Geometric objects, Loose Substances, Porous Substances, } \\
\text { Particles, Plasma, Chemical Compounds }\end{array}$ \\
\hline Energy & $\begin{array}{l}\text { Forces, Motion, Deformation, Thermal Energy, Mechanical and Sound Waves, } \\
\text { Electric Field, Magnetic Field, Nuclear Energy, Electromagnetic Waves or Light }\end{array}$ \\
\hline Parameters & $\begin{array}{l}\text { Surfaces Parameters, Geometric Parameters, Fluids Parameters, Concentration } \\
\text { Parameters, Forces Parameters, Motion and Vibration Parameters, Thermal } \\
\text { Parameters, Electric field Parameters, Magnetic field Parameters, etc. }\end{array}$ \\
\hline
\end{tabular}

\subsection{Function Model}

Products are defined by the overall functions. The overall function can be broken down into several sub-functions. The aggregation of functions and their relations is called function model. There are two types of relations between functions.

One is the relation generated through function decomposition, called hierarchical relation, which illustrates the hierarchical relationships among sub-functions, and discovers the hierarchical design purposes. There are two logical relationships: conjunction and disjunction.

- Conjunction structure: the parent function is achieved by several corporate subfunctions, as shown in Fig. 3(a).

- Disjunction structure: the parent function is achieved by only one of several subfunctions according to condition, as shown in Fig. 3(b).

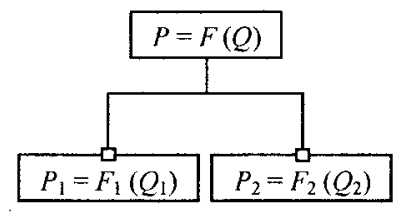

(a) Conjunction structure

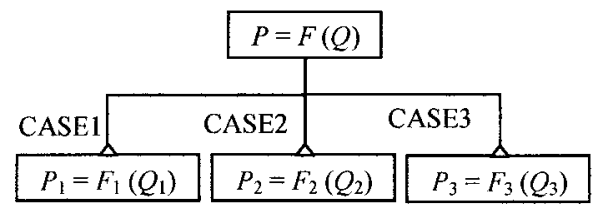

(b) Disjunction structure

Fig. 3. The hierarchical relationships among sub-functions

Another is lateral relation if the input flow of one function is the output flow of the other, which indicates the interaction relationships among active sub-functions at the related level of functional model, reflecting logically and physically possible or useful associations of the sub-functions. There are four logical relationships: serial, parallel, ring and control.

- Serial structure: the parent function is achieved by sub-functions occurring in sequence, as shown in Fig. 4(a).

- Parallel structure: the parent function is achieved by sub-functions happening at the same time, as shown in Fig. 4(b).

- Ring structure: the parent function is achieved by sub-functions in which the input of a former function is the output of a latter function, as shown in Fig. 4(c).

- Control structure: the parent function is achieved by several sub-functions in which a function is controlled by other function, as shown in Fig. 4(d). 


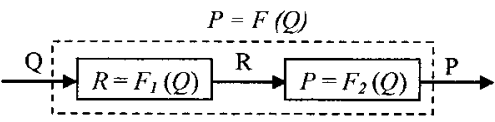

(a) Serial structure $P=F(Q)$

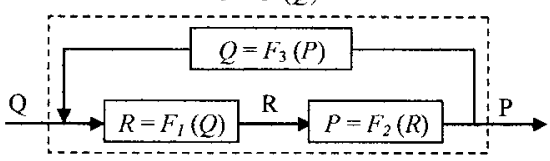

(c) Ring structure

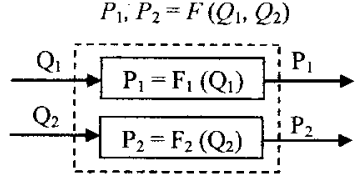

(b) Parallel structure $P=F\left(Q_{l}, Q_{2}\right)$

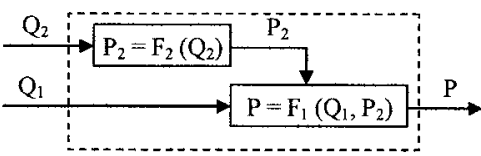

(d) Control structure

Fig. 4. The lateral relationships among sub-functions

\section{Structure}

In conceptual design structure is not concrete structure, but principle structure, which specifies what elements the structure is composed of, what the attributes of the elements are and how they are related.

Any structure is composed of elements. An element in the structure that cannot be divided is called a primitive element. A primitive element can be either a physical or logical entity. Some elements group together and form a sub-structure or structural element that has well-defined characteristics. The interactions of elements are physical interconnection using topological or geometrical data and logical processes.

Structure is a physical embodiment of effect and the change of structure from one state to another must be caused directly or indirectly by effects. By means of patent analysis, the structures, which show how the effect is used for the performance of function transitions, can be acquired. A structure contains structural feature, such as what elements the design is composed of, what the attributes of the elements are and how they are related. An effect can have varied realizing structure, and a structure can be used for varied effects.

\section{Functional Design Based on Effect}

The process of functional design can be seen as transforming a functional representation to a design description or physical representation through function, effect and Structure, as shown in Fig. 5.

- Design begins with the analysis of functional requirements (Q), and then determines the overall function of product $(f)$ in function set.

- According to the inputs and outputs of function, the effect chain can be set up based on effect modes.

- The four logical relationships: serial, parallel, ring and control in effect chain are consistent with them in function model. By the mapping relationship between effect and function, the effects in chain are replaced by relevant function.Thus the 
relationship among sub-functions can be confirmed and the function model is constructed.

- The function model algebra system and the Boolean algebra system are isomorphic, so the function model can be simplified by Boolean calculation of sub-functions.

- By the mapping relationship between effect and structure, each sub-function in function model has its structure, and the product structure is established.

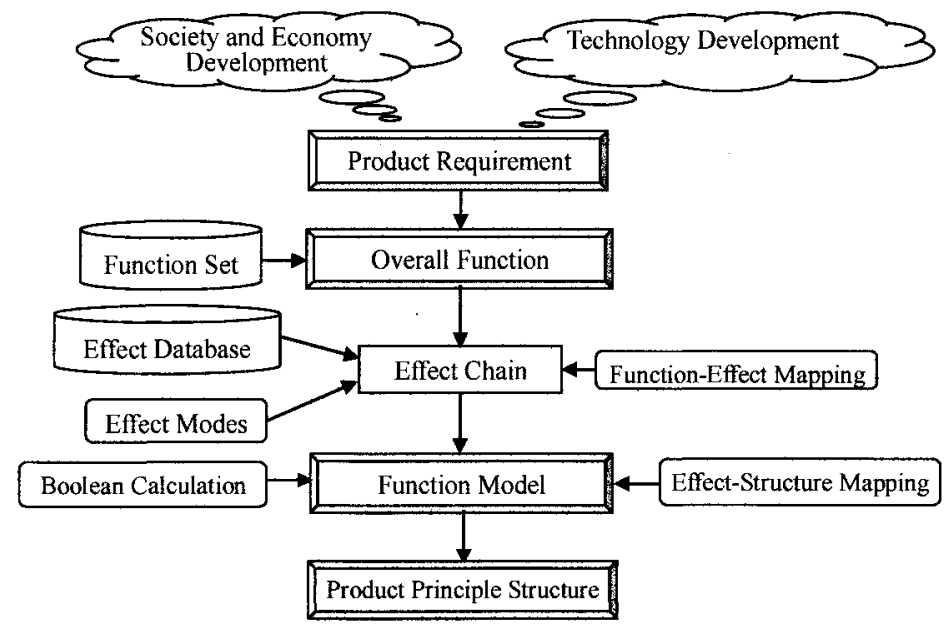

Fig. 5. Functional design process model based on effect

Based on the functional design process, the computer-aided functional design Software (Effect for Functional Design, E2F Design) (in Chinese) is developed by cobweb model and v-model process of software development in Fusion, and is further built by using $\mathrm{VC}++$ and $\mathrm{XML}$. This platform can support function-oriented design in a smooth way.

\section{Case Study}

Pill is a kind of good form of Chinese traditional medicine, but it can not be produced by Western medicine facility for its process and physics characteristic. The present condition is long process, high energy consume and great labor intension, so it is important to develop continue forming and shorten process to meet the need of modern times.

The overall function of granulator system can be modeled in Fig. 6, whose inputs are powder (medicinal powder) and liquid (cementing liquid), and whose outputs are sphericity and particle (pill). According to the known inputs and outputs, search for the effects in effect database of E2F Design software. The effects can be automatically linked into effect chains by using effect modes. Fig. 7 shows the part of effect chains of granulator system. Fig. 8 shows the function model of granulator 
system based on effect chain in Fig. 7(c). Fig. 9 shows the principle structure of granulator system.

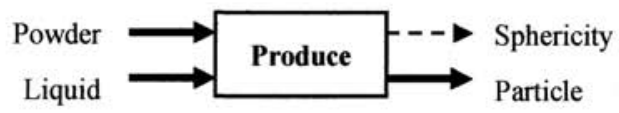

Fig. 6. Overall function of granulator system
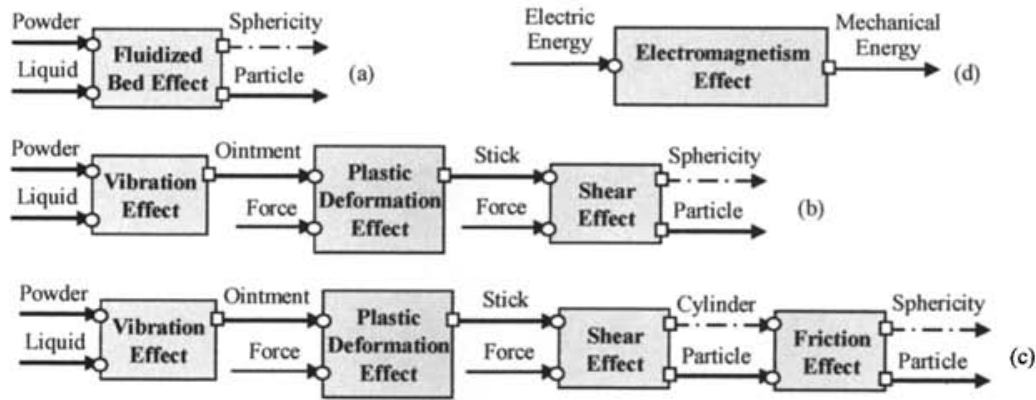

Fig. 7. Effect chains of granulator system

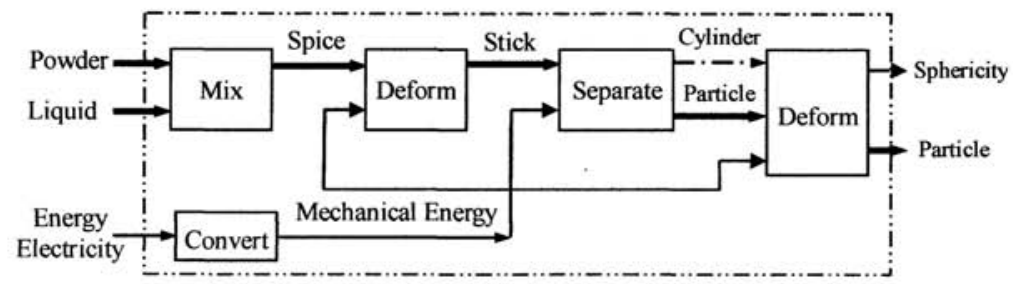

Fig. 8. Function model of granulator system

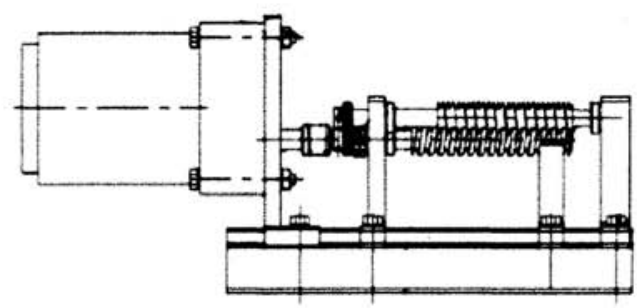

Fig. 9. Principle structure of granulator system 


\section{Conclusion}

Functional design, which includes function understanding, function recognition, function representation, function modeling and function-to-structure mapping, plays the central role in ensuring design quality and product innovation. How to reason effectively and describe completely function model and how to map from function to structure are crucial issues in conceptual design phase.

This paper proposes a product functional design approach based on effect by the followings:

- The multi-pole effect model and effect modes for effect chain are created to extend effect in TRIZ;

- The functions and flows in TRIZ are reclassified and the standard function set is presented;

- The combinative relationships among functions are discussed and the function modeling based on effect is proposed;

- The functional design process model for function modeling and function-tostructure mapping is proposed, and the computer-aided functional design software is developed.

- A design example for conceptual design of air exhaust valve in car is presented to demonstrate the proposed functional design process and prove that the method is feasible.

\section{Acknowledgements}

The research is supported in part by the Chinese Natural Science Foundation under Grant Numbers 50675059 and Chinese national 863 planning project under Grant Number 2006AA04Z109. No part of this paper represents the views and opinions of any of the sponsors mentioned above.

\section{References}

1. L. Qian, and J.S. Gero, Function-Behavior-Structure Paths and their Role in Analogybased Design, AIEDAM, 10, 289-312 (1996).

2. G. Prabhakar, and A. Goel, A functional modeling for adaptive design of devices in new environments, Artificial Intelligence in Engineering Journal (Special Issue), 12(4), 417444 (1998).

3. Y. Shimomura, H. Takeda and et al., Representation of Design Object based on the Functional Evolution Process Model. DTM'95-ASME (1995).

4. B. Chandrasekaran and H. Kaindl, Representing Functional Requirements and Usersystem Interactions. AAAI Workshop on Modeling and Reasoning about Function, pp.7884 (1996).

5. Y. Umeda and T. Tomiyama, FBS Modeling: Modeling Scheme of Function for Conceptual Design, Workshop on Qualitative Reasoning about Phys. Systems, Amsterdam, pp.271-278 (1995).

6. G. Pahl and W. Beitz, Engineering Design - A Systematic approach, The 2nd Edition, Springer-Verlag London (1996). 
7. J. Collins, B. Hagan and H. Bratt, The Failure-Experience Matrix - a Useful Design Tool, Transactions of the ASME, Series B, Journal of Engineering in Industry, 98, 1074-1079 (1976).

8. G. Altshuller, Creativity as an Exact Science, Gordon and Branch Publishers, Luxembourg (1984).

9. J. Malmqvist, R. Axelsson, and M. Johansson, Comparative Analysis of the Theory of Inventive Problem Solving and the Systematic Approach of Pahl and Beitz, Proceedings of the 1996 ASME Design Engineering Technical Conference and Computers in Engineering Conference, Irvine, CA (1996).

10. N.P. Suh, The Principle of Design. New York: Oxford University Press (1990).

11. R.H. Sturges, Computational model for conceptual design based on extended function logic, Artificial Intelligent for Engineering Design Analysis and Manufacturing, 10, 255 274 (1996).

12. B.S. Chen, Characterization and Representation of Functional Requirements and Functional Tolerancing for Concurrent Design and Manufacturing [Ph. D. Thesis], Ohio State University, USA, (1994).

13. Y. Umeda, M. Ishii, M. Yoshioka, et al., Supporting conceptual design based on the function-behavior-state modeler, Artificial Intelligence for Engineering Design, Analysis and Manufacturing: Aiedam, 10 (4), 275-288 (1996).

14. Y.M. Deng, S.B. Tor, and G.A. Britton, Abstracting and exploring functional design information for conceptual product design, Engineering with Computers, 16, 36-52 (2000).

15. D. Tate, A Roadmap for Decomposition: Activities, Theories, and Tools for System Design [Ph.D Thesis], Department of mechanical Engineering, MIT, Cambridge, MA USA, (1999).

16. G. Altshuller, The Innovation Algorithm, TRIZ, Systematic Innovation and Technical Creativity, Technical Innovation Center, INC, Worcester (1999).

17. F. Gregory, Classifying the Technical Effects, TRIZ Journal, 3, http://www.triz journal.com (1998).

18. Kalevi R. Eleven Uses of Effects. TRIZ Journal, 9, http://www.triz-journal.com (1998).

19. B. Bohuslav, M. Darrell and J. Pavel, Case Studies in TRIZ: A Novel Heat Exchanger, TRIZ Journal, 12, http://www.triz-journal.com (1999).

20. R. Tan, Innovation Design-TRIZ: Theory of Innovative Problem Solving, China Mechanic Press (in Chinese) (2002). 